Article

\title{
The Seawall Bargaining Game
}

\section{Rémy Delille and Jean-Christophe Pereau *}

GREThA, Université de Bordeaux, Avenue Léon Duguit, 33608 Pessac Cedex, France;

E-Mail: remy.delille@u-bordeaux.fr

* Author to whom correspondence should be addressed;

E-Mail: jean-christophe.pereau@u-bordeaux.fr; Tel.: +33-(0)5-56-84-29-66.

Received: 24 April 2014; in revised form: 9 June 2014 / Accepted: 19 June 2014 /

Published: 24 June 2014

\begin{abstract}
Agents located from downstream to upstream along an estuary and exposed to a flooding risk have to invest in facilities like a seawall (or dike). As the benefits of that local public good increase along the estuary, upstream agents have to bargain for monetary compensation with the most downstream agent in exchange for more protection effort. The paper analyses different bargaining protocols and determines the conditions under which agents are better off. The results show that upstream agents are involved in a chicken game when they have to bargain with the most downstream agent.
\end{abstract}

Keywords: alternating-offer model; chicken game; public good; sea-flood; seawall

JEL classifications: $\quad$ C78

\section{Introduction}

Hirshleifer [1] shows with his "Anarchia Island" fable that citizens have successfully agreed to build seawalls (or dikes) to protect themselves from storms threatening to flood the coastline despite the weakest-link structure of that local public good. The seawall is known as a particular public good in which the level of effective protection for the whole island depends on the citizen who has constructed the lowest seawall. This paper analyses a similar problem of flood-protection when agents are located subsequently from downstream to upstream along an estuary (exposed to flooding risk). In that case, flood protection does not only consist in building the highest seawall, it also requires the construction of other facilities, such as a first seawall to break the wave, the use of wetland as flood water retention 
land, a network of small rivers to control the flood or a second seawall. This estuarine geography feature implies that when the sea enters, the protection effort implemented by an agent will be a public good for all of the agents who are located upstream from him. At the two ends of the spectrum, the most downstream agent (the closest to the sea) gets nothing from the public good, while the most upstream agent benefits from the efforts made by all of his downstream neighbors. Thus, our seawall bargaining game is no longer a problem of public good with a weakest-link aggregation technology, but a public good with positive externalities that are increasing along the estuary. Since the benefit of the public good an agent gets depends on where he is located, it makes cooperation harder to achieve. To ensure a high level of effort from the most downstream agent, upstream agents have to make monetary transfers. To model such negotiations, the most obvious approach is to base the analysis on what is know as bargaining theory with the Rubinstein alternating-offers model. Rubinstein [2] describes the process through which negotiating agents can reach an agreement. The agent opening the negotiations makes an offer. The other agent can either accept the offer, in which case the negotiation ends, or reject it and make a counter-offer, which may also be accepted or rejected with a new counter-offer. In our framework, an offer will cover two variables related to the effort of sea-flood protection and a monetary transfer.

The seawall bargaining game can be modeled as a particular case of global public good in which all agents benefit from the action of the other players, whatever their location. In the literature on international environmental agreements, for instance, results show that for identical agents, only a very small number of players will form a coalition. Seminal papers using this approach are Carraro and Siniscalco [3] and Barrett [4], and a survey can be found in Finus [5]. However, this approach often ignores the negotiating process in terms of offers and counter-offers that characterize all negotiations between self-interested agents. Our framework shares, in common with the "sharing river" model, the downstream/upstream agent structure in which downstream agents create negative externalities to upstream agents. This literature is based on cooperative game theory and, more precisely, on the core (see Ambec and Sprumont [6] and Beal et al. [7] for a survey). The objective is to set up a burden-sharing rule able to favor the cooperation of all. The sharing rule aims at preventing any individual agent, but also any sub-group of agents, from having no incentive to leave the agreement. It is crucial to thoroughly describe the bargaining process that designs the most likely burden-sharing rule (Carraro et al., [8]). Wang [9] analyses a market-based approach using trading water rights, but restricts the bilateral trading to neighboring agents. Houba [10] computes the Rubinstein bargaining solution in a bilateral case, and Houba et al. [11] apply the asymmetric Nash bargaining solution. Our framework also assumes Rubinstein bargaining when several agents are involved and analyses the different bargaining protocols that are preferred by agents.

The rest of the paper is organized as follows. We start in Section 2 by analyzing the model and the cooperative and non-cooperative outcomes. Section 3 is devoted to the analysis of single and double negotiations. In Section 4, a specific example shows the main results of the paper.

\section{The Model}

Considering a three-agent framework is enough to put forward the main results of the paper. Every agent is located in a lexicographic ordering. Agent 1 is the most downstream agent and agent 3 is the 
most upstream agent. We note $e_{i}$, the effort of protection realized by agent $i$; their respective payoffs are defined as the difference between the concave benefit function $\left(B_{i}^{\prime}>0\right.$ and $\left.B_{i}^{\prime \prime}<0\right)$ and the convex cost function $\left(C_{i}^{\prime}>0\right.$ and $\left.C_{i}^{\prime \prime}>0\right)$ :

$$
\begin{aligned}
\pi_{1}\left(e_{1}\right) & =B_{1}\left(e_{1}\right)-C_{1}\left(e_{1}\right) \\
\pi_{2}\left(e_{1}, e_{2}\right) & =B_{2}\left(e_{1}+e_{2}\right)-C_{2}\left(e_{2}\right) \\
\pi_{3}\left(e_{1}, e_{2}, e_{3}\right) & =B_{3}\left(e_{1}+e_{2}+e_{3}\right)-C_{3}\left(e_{3}\right)
\end{aligned}
$$

Equations (2) and (3) show that in addition to their own efforts, agent 2 benefits from the effort made by agent 1 and agent 3 from the efforts of both agents 1 and 2 . On the opposite side, agent 1 gets nothing from the others. Costs are private.

Cooperative outcome: The cooperative solution is given by the program:

$$
\max _{e_{i}} \sum_{i=1}^{3} \pi_{i}(\mathbf{e})
$$

Effort levels are solution of:

$$
\begin{aligned}
B_{1}^{\prime}\left(e_{1}\right)+B_{2}^{\prime}\left(e_{1}+e_{2}\right)+B_{3}^{\prime}\left(e_{1}+e_{2}+e_{3}\right) & =C_{1}^{\prime}\left(e_{1}\right) \\
B_{2}^{\prime}\left(e_{1}+e_{2}\right)+B_{3}^{\prime}\left(e_{1}+e_{2}+e_{3}\right) & =C_{2}^{\prime}\left(e_{2}\right) \\
B_{3}^{\prime}\left(e_{1}+e_{2}+e_{3}\right) & =C_{3}^{\prime}\left(e_{3}\right)
\end{aligned}
$$

It returns a unique vector of efforts $\mathbf{e}^{c}=\left\{e_{1}^{c}, e_{2}^{c}, e_{3}^{c}\right\}$. An additional unit of effort by agent $i$ exerts an additional benefit for him and for agents upstream of his position. At the equilibrium, the marginal cost of that unit equalizes the sum of his marginal benefit and the marginal benefit of the upstream agents.

Non-cooperative outcome: Whenever agents act in a non-cooperative way, the optimality conditions are:

$$
\begin{aligned}
B_{1}^{\prime}\left(e_{1}\right) & =C_{1}^{\prime}\left(e_{1}\right) \\
B_{2}^{\prime}\left(e_{1}+e_{2}\right) & =C_{2}^{\prime}\left(e_{2}\right) \\
B_{3}^{\prime}\left(e_{1}+e_{2}+e_{3}\right) & =C_{3}^{\prime}\left(e_{3}\right)
\end{aligned}
$$

and give the non-cooperative efforts $\mathbf{e}^{n c}=\left\{e_{1}^{n c}, e_{2}^{n c}, e_{3}^{n c}\right\}$. Each agent equalizes his marginal benefit to his marginal cost.

The differences between the two systems of optimality conditions, (4)-(6) and (7)-(9), suggest that agents 1 and 2 will realize more effort in the cooperative case than in the non-cooperative case, because they take into account the positive impact of their action on agents located upstream. Indeed for agent 2 , two effects are at stake. The additional effort of agent 1 in the cooperative case reduces agent 2's incentives to do likewise, but due to the positive impact that 2 exerts on agents upstream, agent 2 tends to increase his effort in the cooperative case. This latter effect dominates the former, since $e_{i}^{n c}<e_{i}^{c}$ for both agents $i=1,2$. However, this is the opposite for the most upstream agent. His effort is reduced in the cooperative case, since he benefits from the effort made by the previous downstream agents, leading to $e_{3}^{c}<e_{3}^{n c}$. The aggregate effort is higher in the cooperative case than in the non-cooperative case. 
In terms of payoffs, agents 2 and 3 are better off in the cooperative case, unlike the most upstream agent, whose payoff only depends on his effort. It turns that agent 1 has to make a higher effort in the non-cooperative case, and this substantially reduces his payoff. It yields $\pi_{i}^{c}>\pi_{i}^{n c}$ for $i=2,3$ but $\pi_{1}^{c}<\pi_{1}^{n c}$. This is the main feature of the estuarine geography. The dominant strategy of agent 1 is to implement his non-cooperative effort regardless of the action of the upstream agents. Hence, moving from the non-cooperative case to the cooperative case will never be profitable for the most downstream agent, even if the aggregate payoff in the cooperative case is higher than in the non-cooperative case.

\section{Negotiation Protocols}

Negotiation is a solution to improve the payoffs of all of the agents and, in particular, for the most downstream agent, who has no incentive to increase his effort. The negotiation can take place between agents over an extra amount of effort that an agent will implement in exchange for a monetary transfer or compensation. However, several protocols must be considered given the multiplicity of pairs of agents. Bargaining between two agents consists in an effort and a transfer, denoted hereafter by $o_{i}=\left(e_{i}, \tau_{i j}\right)$ for $i=1,2 . j \neq i$. Negotiations take place under perfect information. It is assumed that there is no authority able to impose an agreement or a particular protocol on private agents. The simplest negotiation only concerns two players, either agent 1 with 2 or 3 or between 2 and 3 . In all cases, agent 3 maximizes his individual payoff by setting his effort $e_{3}$. Payoffs are denoted by $V_{1}\left(e_{1}\right)=B_{1}\left(e_{1}\right)-C_{1}\left(e_{1}\right), V_{2}\left(e_{1}, e_{2}\right)=$ $B_{2}\left(e_{1}+e_{2}\right)-C_{2}\left(e_{2}\right), V_{3}\left(e_{1}, e_{2}, e_{3}\right)=B_{3}\left(e_{1}+e_{2}+e_{3}\right)-C_{3}\left(e_{3}\right)$.

In a three-agent case, the general bargaining framework is given by the following net payoff function:

$$
\begin{aligned}
\pi_{1}\left(o_{1}, t\right) & =\pi_{1}^{n c}+\delta_{1}^{t}\left(V_{1}\left(e_{1}\right)-\pi_{1}^{n c}+\tau_{12}+\tau_{13}\right) \\
\pi_{2}\left(o_{1}, o_{2} ; t\right) & =\pi_{2}^{n c}+\delta_{2}^{t}\left(V_{2}\left(e_{1}, e_{2}\right)-\pi_{2}^{n c}-\tau_{12}+\tau_{23}\right) \\
\pi_{3}\left(o_{1}, o_{2}, e_{3} ; t\right) & =\pi_{3}^{n c}+\delta_{3}^{t}\left(V_{3}\left(e_{1}, e_{2}, e_{3}\right)-\pi_{3}^{n c}-\tau_{13}-\tau_{23}\right)
\end{aligned}
$$

where $0<\delta_{i}<1$ stands for the discount factor of agent $i$.

Proposition 1. When the negotiation is over a simple pair $\left(e_{i}, \tau_{i j}\right)$ of effort and transfer between agents $i$ (the proposer) and $j$ (the responder) for $i=1,2, j=2,3, j \neq i$ and $j \neq k$, the optimal vector of efforts e satisfies:

$$
\begin{aligned}
B_{i}^{\prime}(\mathbf{e})+B_{j}^{\prime}(\mathbf{e}) & =C_{i}^{\prime}\left(e_{i}\right) \\
B_{j}^{\prime}(\mathbf{e}) & =C_{j}^{\prime}\left(e_{j}\right) \\
B_{k}^{\prime}(\mathbf{e}) & =C_{k}^{\prime}\left(e_{k}\right)
\end{aligned}
$$

and the associated payoffs are:

$$
\begin{aligned}
& \pi_{i}^{*}(\mathbf{e})=\pi_{i}^{n c}+\frac{\left(1-\delta_{j}\right)}{\left(1-\delta_{i} \delta_{j}\right)} \pi \\
& \pi_{j}^{*}(\mathbf{e})=\pi_{j}^{n c}+\frac{\delta_{j}\left(1-\delta_{i}\right)}{\left(1-\delta_{i} \delta_{j}\right)} \pi \\
& \pi_{k}^{*}(\mathbf{e})=\pi_{k}^{n c}+\left(V_{k}^{*}-\pi_{k}^{n c}\right)
\end{aligned}
$$

where $\pi=V_{i}^{*}-\pi_{i}^{n c}+V_{j}^{*}-\pi_{j}^{n c}>0$ stands for the created surplus. 
Proof. See the proof in Section 6.1.

Simple negotiations refer to the following cases: 1 bargains with 3 over $\left(e_{1}, \tau_{13}\right)$; 1 bargains with 2 over $\left(e_{1}, \tau_{12}\right)$ and 2 bargains with 3 over $\left(e_{2}, \tau_{23}\right)$. In each case, the outsider of the negotiation acts non-cooperatively. When 2 or 3 bargains with 1 , the negotiation implies a higher effort for $1, e_{1}^{c}>e_{1}^{*}>$ $e_{1}^{n c}$ and lower efforts for 2 or $3 e_{j}^{c}<e_{j}^{*}<e_{j}^{n c}, j=2,3$. When the negotiation is between 2 and 3,2 increases his effort at the expense of 3 , such that $e_{2}^{*}>e_{2}^{c}>e_{2}^{n c}$ and $e_{3}^{c}<e_{3}^{*}<e_{3}^{n c}$, while the effort of 1 remains unchanged $e_{1}^{*}=e_{1}^{n c}$. When agents bargain, they get a share of the surplus that they have created, while the outsider acts as a free-rider and benefits from the public good, except when the outsider is the most downstream agent, since his payoff equals his non-cooperative outcome. This particular case refers to the maximization of the aggregate payoff under the constraint that the most downstream agent gets his non-cooperative payoff. In the limit when the time between bargaining rounds vanishes $\delta=\delta_{i} \rightarrow 1 \forall i$, the created surplus is shared equally. In that case, the Rubinstein solution converges to the Nash solution. It turns out that agent 2 is better off when 3 bargains with 1, and by symmetry, 3 is better off when 2 bargains with 1 .

Expecting that the created surplus can be higher when all agents are at the negotiation table, we assume that the three agents negotiate in two bilateral negotiations. This structure ensures the uniqueness of the subgame perfect equilibrium (SPE) ${ }^{1}$. Two cases are considered. Firstly, 3 bargains twice over $\left(e_{1}, \tau_{13}\right)$ with 1 and over $\left(e_{2}, \tau_{23}\right)$ with 2 (setting $\left.\tau_{12}=0\right)$. Secondly, 2 bargains twice over $\left(e_{1}, \tau_{12}\right)$ with 1 and over $\left(e_{2}, \tau_{23}\right)$ with 3 (setting $\tau_{13}=0$ ).

The first negotiation yields the following proposition.

Proposition 2. When 3 bargains as a proposer with 1 over $\left(e_{1}, \tau_{13}\right)$ and with 2 over $\left(e_{2}, \tau_{23}\right)$, the Rubinstein bargaining solution shows that:

1. The optimal vector of efforts e satisfies:

$$
\begin{aligned}
B_{1}^{\prime}\left(e_{1}\right)+B_{3}^{\prime}\left(e_{1}+e_{2}+e_{3}\right) & =C_{1}^{\prime}\left(e_{1}\right) \\
B_{2}^{\prime}\left(e_{1}+e_{2}\right)+B_{3}^{\prime}\left(e_{1}+e_{2}+e_{3}\right) & =C_{2}^{\prime}\left(e_{2}\right) \\
B_{3}^{\prime}\left(e_{1}+e_{2}+e_{3}\right) & =C_{3}^{\prime}\left(e_{3}\right)
\end{aligned}
$$

2. Equilibrium payoffs after transfers are:

$$
\begin{aligned}
& \pi_{i}^{*}=\pi_{i}^{n c}+\frac{\delta_{i}\left(1-\delta_{j}\right)\left(1-\delta_{3}\right)}{\eta} \pi, i=1,2 \text { and } j \neq i \\
& \pi_{3}^{*}=\pi_{3}^{n c}+\frac{\left(1-\delta_{1}\right)\left(1-\delta_{2}\right)}{\eta} \pi
\end{aligned}
$$

where $\pi=V_{1}^{*}-\pi_{1}^{n c}+V_{2}^{*}-\pi_{2}^{n c}+V_{3}^{*}-\pi_{3}^{n c}>0$ stands for the created surplus and $\eta=$ $\left(1-\delta_{1} \delta_{3}\right)\left(1-\delta_{2} \delta_{3}\right)-\delta_{1} \delta_{2}\left(1-\delta_{3}\right)^{2}>0$

1 As shown by Shaked and reported by Sutton [12], the use of Rubinstein model in a multilateral bargaining framework may yield multiple equilibria under the unanimity rule. 
Proof. See the proof in Section 6.2.

The second negotiation yields the following proposition.

Proposition 3. When 2 bargains simultaneously as a proposer with 1 over $\left(e_{1}, \tau_{12}\right)$ and with 3 over $\left(e_{2}, \tau_{23}\right)$, the Rubinstein bargaining solution shows that:

1. The optimal vector of efforts e satisfies:

$$
\begin{aligned}
B_{1}^{\prime}\left(e_{1}\right)+B_{2}^{\prime}\left(e_{1}+e_{2}\right) & =C_{1}^{\prime}\left(e_{1}\right) \\
B_{2}^{\prime}\left(e_{1}+e_{2}\right)+B_{3}^{\prime}\left(e_{1}+e_{2}+e_{3}\right) & =C_{2}^{\prime}\left(e_{2}\right) \\
B_{3}^{\prime}\left(e_{1}+e_{2}+e_{3}\right) & =C_{3}^{\prime}\left(e_{3}\right)
\end{aligned}
$$

2. Equilibrium payoffs (after transfers) are:

$$
\begin{gathered}
\pi_{i}^{*}=\pi_{i}^{n c}+\frac{\delta_{i}\left(1-\delta_{2}\right)\left(1-\delta_{j}\right)}{\phi} \pi, i=1,3 \text { and } j \neq i \\
\pi_{2}^{*}=\pi_{2}^{n c}+\frac{\left(1-\delta_{1}\right)\left(1-\delta_{3}\right)}{\phi} \pi \\
\text { where } \pi=V_{1}^{*}-\pi_{1}^{n c}+V_{2}^{*}-\pi_{2}^{n c}+V_{3}^{*}-\pi_{3}^{n c}>0 \text { stands for the created surplus and } \\
\phi=\left(1-\delta_{1} \delta_{2}\right)\left(1-\delta_{2} \delta_{3}\right)-\delta_{1} \delta_{3}\left(1-\delta_{2}\right)^{2}>0 .
\end{gathered}
$$

Proof. See the proof in Section 6.3.

In both negotiations, agents 1 and 2 will increase their efforts, such that $e_{1}^{c}>e_{1}^{*}>e_{1}^{n c}$ and $e_{2}^{*}>e_{2}^{c}>e_{2}^{n c}$, implying a decrease in the effort for the most upstream agent $e_{3}^{n c}>e_{3}^{*}>e_{3}^{c}$ with respect to his non-cooperative effort. Agents get a share of the surplus that they have created, but the agent who is always the proposer has a first mover advantage. However, in the limit associated with instantaneous negotiations $\delta=\delta_{i} \rightarrow 1 \forall i$, they all get one third of the surplus. The two negotiations show that agents are better off than with the non-cooperative case $\pi_{1}^{*}>\pi_{1}^{n c}>\pi_{1}^{c}$ and $\pi_{i}^{c}>\pi_{i}^{*}>\pi_{i}^{n c}$ for $i=2,3$. However, in both cases, the size of the created surplus is different, since equilibrium efforts did not satisfy the same optimality conditions.

\section{Results}

With the will to make tractable comparisons between the negotiation protocols, we assume that agents have identical benefit and cost functions, but differ in their location along the estuary:

$$
\begin{aligned}
& B(z)=a z-\frac{b}{2} z^{2}, a, b>0 \\
& C(z)=\frac{c}{2} z^{2}, c>0
\end{aligned}
$$

It is also assumed that $\delta=\delta_{i} \rightarrow 1 \forall i$. Based on Tables 2 to 4 in Section 6.4, which summarize the outcome for all bargaining protocols under the previous assumptions, several assertions can be drawn from the results. 
Result 1: The cooperative outcome that gives the highest aggregate effort and payoff cannot be reached by a particular negotiation protocol. This result comes from the structure of the model. The dominant strategy of the most downstream agent is to act non-cooperatively. It turns out that an extra effort will always decrease his payoff. Only a constrained cooperative solution can be obtained. This solution consists in maximizing the aggregate outcome under the constraint than the most downstream agent gets his non-cooperative outcome. However, results show that agents are always better off when negotiations over efforts and a set of transfers exist.

Result 2: In a single negotiation, agent 2 (respectively, 3) prefers to free ride and be the outsider of a negotiation between 1 and 3 (respectively, 2). This conflict of interest between agents 2 and 3 comes from the presence of the public good. Each agent would rather benefit from the efforts realized by the other at no cost, as shown in Table 1. The three-agent seawall bargaining game can be summarized in normal form where the space of strategies of each agent consists in either the acceptance (A) or the refusal (R) of negotiations. $S_{i}=\{A, R\}$ for $i=\{1,2,3\}$. Notation $i \leftrightarrow j$ means that $i$ negotiates with $j$. It follows:

Table 1. The seawall bargaining game.

\begin{tabular}{c|c|c}
\hline \multicolumn{3}{c}{$\mathbf{S}_{1}=\mathbf{A}$} \\
\hline $2 \backslash 3$ & $S_{3}=A$ & $S_{3}=R$ \\
\hline$S_{2}=A$ & $\pi_{1}^{\{2 \leftrightarrow 1,3\}}, \pi_{2}^{\{2 \leftrightarrow 1,3\}}, \pi_{3}^{\{2 \leftrightarrow 1,3\}}$ & $\pi_{1}^{\{1 \leftrightarrow 2\}}, \pi_{2}^{\{1 \leftrightarrow 2\}}, \pi_{3}^{F}$ \\
\hline$S_{2}=R$ & $\pi_{1}^{\{1 \leftrightarrow 3\}}, \pi_{2}^{F}, \pi_{3}^{\{1 \leftrightarrow 3\}}$ & $\pi_{1}^{n c}, \pi_{2}^{n c}, \pi_{3}^{n c}$ \\
\hline \multicolumn{2}{|c}{$\mathbf{S}_{1}=\mathbf{R}$} \\
\hline $2 \backslash 3$ & $S_{3}=A$ & $S_{3}=R$ \\
\hline$S_{2}=A$ & $\pi_{1}^{n c}, \pi_{2}^{\{2 \leftrightarrow 3\}}, \pi_{3}^{\{2 \leftrightarrow 3\}}$ & $\pi_{1}^{n c}, \pi_{2}^{n c}, \pi_{3}^{n c}$ \\
\hline$S_{2}=R$ & $\pi_{1}^{n c}, \pi_{2}^{n c}, \pi_{3}^{n c}$ & $\pi_{1}^{n c}, \pi_{2}^{n c}, \pi_{3}^{n c}$ \\
\hline
\end{tabular}

We consider that if agent 1 refuses to bargain while the strategies of 2 and 3 are to accept, then 2 and 3 bargain between themselves. Then, the seawall bargaining game shows that there exists two Nash equilibria in pure strategies $\left(S_{1}, S_{2}, S_{3}\right)=(A, R, A)$ and $(A, A, R)$. In both cases, agent 1 always accepts to negotiate, since his payoff is higher than his non-cooperative payoff, $\pi_{1}^{\{1 \leftrightarrow 2\}}>\pi_{1}^{n c}$ and $\pi_{1}^{\{1 \leftrightarrow 3\}}>\pi_{1}^{n c}$. Concerning the behavior of agents 2 and 3, they are better off when they act as free riders, $\pi_{i}^{F}>\pi_{i}^{\{2 \leftrightarrow 1,3\}}$ and $\pi_{i}^{\{1 \leftrightarrow i\}}>\pi_{i}^{n c}$ for $i=2,3$. The structure of the seawall bargaining game corresponds to a chicken game, as in Carraro and Siniscalco [3]. agent 2 (respectively, 3) prefers that his opponent bargains with 1 and benefits from the outcome of the negotiation without bearing any cost. 
In double negotiation, the conflict is over the position of the proposer, but this is a direct consequence of the Rubinstein alternating offer model. When the time between bargaining rounds vanishes, this first mover advantage disappears.

Result 3: It is socially optimal to ask agent 2 to manage the two negotiations with the most downstream and the most upstream agents. It can be shown that the surplus in double negotiation is higher than single negotiation. The size of the created surplus increases with the number of negotiators. Moreover, results show that the surplus is higher when 2 bargains twice instead of 3 . A negotiation between 2 and 1 yields a higher effort of agent 1 in comparison with a negotiation between 3 and 1 . This higher effort increases the benefit of the public good for 2 and 3. When 3 bargains twice, efforts made by 1 or 2 are substitutable, leading 3 to bargain a higher effort with 2 than with 1 . This result can be generalized to $n$ agents. Individual payoffs will be better off for all agents when $n$ negotiates with $n-1$, $n-1$ with $n-2, \ldots$, until 2 with 1.

\section{Conclusions}

Hirshleifer [1] shows that cooperation over the building of a seawall can be achieved even if the seawall is known as a weakest-link public good. The seawall example has been revisited in another geographic structure where agents are located from downstream to upstream and have to decide their effort to protect themselves from sea floods. This feature implies that the benefit of the public good increases along an estuary. In a simplified three-agent framework, our results show that there does not exist a bargaining protocol that can be preferred by all of the agents. Agents located after the most downstream agent always prefer free riding rather than entering in single negotiations over an additional effort from the most downstream agent. This case refers to a chicken game. When the negotiation involves all of the agents, our results show that it is more profitable for society to give the right to the agent located in the middle of the estuary to conduct negotiations with both the most downstream and the most upstream agent.

\section{Proofs}

\subsection{Proposition 1}

Consider the single negotiation between 1 and 2 over $\left(e_{1}, \tau_{12}\right)$ with $\tau_{13}=\tau_{23}=0$. agents 1 and 2 make offers and counteroffers over $o_{1}^{(j)}=\left(e_{1}^{(j)}, \tau_{12}^{(j)}\right)$, where $o_{1}^{(j)}$ denotes the offer made by $j=1,2$. The subgame perfect equilibrium (SPE) offers solve the two indifference conditions (Muthoo [13]):

$$
\begin{aligned}
\pi_{1}\left(o_{1}^{(2)}, 0\right) & =\pi_{1}\left(o_{1}^{(1)}, 1\right) \\
\pi_{2}\left(o_{1}^{(1)}, o_{2} ; 0\right) & =\pi_{2}\left(o_{1}^{(2)}, o_{2} ; 1\right)
\end{aligned}
$$

so that each agent is indifferent between accepting the current offer of his opponent and making a counteroffer in the next period. Specifically, the offers satisfy:

$$
\begin{gathered}
V_{1}\left(e_{1}^{(2)}\right)+\tau_{12}^{(2)}=\left(1-\delta_{1}\right) \pi_{1}^{n c}+\delta_{1}\left(V_{1}\left(e_{1}^{(1)}\right)+\tau_{12}^{(1)}\right) \\
V_{2}\left(e_{1}^{(1)}, e_{2}\right)-\tau_{12}^{(1)}=\left(1-\delta_{2}\right) \pi_{2}^{n c}+\delta_{2}\left(V_{2}\left(e_{1}^{(2)}, e_{2}\right)-\tau_{12}^{(2)}\right)
\end{gathered}
$$


The optimal offer of agent 1 to agent $2\left(o_{1}^{(1)}\right)$ has to maximize his payoff under the constraint (11) for 2. Substitute the expression of the transfer $\tau_{12}^{(1)}$; the offer of 1 satisfies:

$$
B_{1}^{\prime}\left(e_{1}\right)+B_{2}^{\prime}\left(e_{1}+e_{2}\right)=C_{1}^{\prime}\left(e_{1}\right)
$$

It can be shown that the optimal offer of 2 to 1 satisfies the same condition. Assume that 1 is the proposer; the equilibrium transfer is $\tau_{12}^{*}=\tau_{12}^{(1)}$ :

$$
\tau_{12}^{*}=-\frac{\left(1-\delta_{1}\right) \delta_{2}}{\left(1-\delta_{1} \delta_{2}\right)}\left(V_{1}^{*}-\pi_{1}^{n c}\right)+\frac{\left(1-\delta_{2}\right)}{\left(1-\delta_{1} \delta_{2}\right)}\left(V_{2}^{*}-\pi_{2}^{n c}\right)
$$

and the equilibrium payoffs:

$$
\begin{aligned}
& \pi_{1}^{*}=\pi_{1}^{n c}+\frac{\left(1-\delta_{2}\right)}{\left(1-\delta_{1} \delta_{2}\right)}\left(V_{1}^{*}-\pi_{1}^{n c}+V_{2}^{*}-\pi_{2}^{n c}\right) \\
& \pi_{2}^{*}=\pi_{2}^{n c}+\frac{\left(1-\delta_{1}\right) \delta_{2}}{\left(1-\delta_{1} \delta_{2}\right)}\left(V_{1}^{*}-\pi_{1}^{n c}+V_{2}^{*}-\pi_{2}^{n c}\right)
\end{aligned}
$$

Agents 2 and 3 maximize their payoffs after transfers, leading to the optimality conditions:

$$
\begin{aligned}
B_{2}^{\prime}\left(e_{1}+e_{2}\right) & =C_{2}^{\prime}\left(e_{2}\right) \\
B_{3}^{\prime}\left(e_{1}+e_{2}+e_{3}\right) & =C_{3}^{\prime}\left(e_{3}\right)
\end{aligned}
$$

The bargaining involving 1 and 3 over $\left(e_{1}, \tau_{13}\right)$ with $\tau_{12}=\tau_{23}=0$ is based on the same method. When the negotiation takes place between 2 and 3, the SPE offers solve the two indifference conditions for 2 $\pi_{2}\left(o_{1}, o_{2}^{(3)} ; 0\right)=\pi_{2}\left(o_{1}, o_{2}^{(2)} ; 1\right)$ and for $3 \pi_{3}\left(o_{1}, o_{2}^{(2)}, e_{3} ; 0\right)=\pi_{3}\left(o_{1}, o_{2}^{(3)}, e_{3} ; 1\right)$.

\subsection{Proposition 2}

3 bargains with 1 over $o_{1}^{(1)}=\left(e_{1}, \tau_{13}\right)$ and with 2 over $o_{2}=\left(e_{2}, \tau_{23}\right)$. The SPE offers solve the two systems of two indifference conditions between 1 and 3 :

$$
\begin{gathered}
V_{1}\left(e_{1}^{(3)}\right)+\tau_{13}^{(3)}=\left(1-\delta_{1}\right) \pi_{1}^{n c}+\delta_{1}\left(V_{1}\left(e_{1}^{(1)}\right)+\tau_{13}^{(1)}\right) \\
V_{3}\left(e_{1}^{(1)}, e_{2}, e_{3}\right)-\tau_{13}^{(1)}-\tau_{23}=\left(1-\delta_{3}\right) \pi_{3}^{n c}+\delta_{3}\left(V_{3}\left(e_{1}^{(3)}, e_{2}, e_{3}\right)-\tau_{13}^{(3)}-\tau_{23}\right)
\end{gathered}
$$

and between 2 and 3 :

$$
\begin{gathered}
V_{2}\left(e_{1}, e_{2}^{(3)}\right)+\tau_{23}^{(3)}=\left(1-\delta_{2}\right) \pi_{2}^{n c}+\delta_{2}\left(V_{2}\left(e_{1}, e_{2}^{(2)}\right)+\tau_{23}^{(2)}\right) \\
V_{3}\left(e_{1}, e_{2}^{(2)}, e_{3}\right)-\tau_{13}-\tau_{23}^{(2)}=\left(1-\delta_{3}\right) \pi_{3}^{n c}+\delta_{3}\left(V_{3}\left(e_{1}, e_{2}^{(3)}, e_{3}\right)-\tau_{13}-\tau_{23}^{(3)}\right)
\end{gathered}
$$

The optimal offer of 1 to $3\left(o_{1}^{(1)}\right)$ maximizes his payoff $\pi_{1}\left(e_{1}\right)=V_{1}\left(e_{1}\right)+\tau_{13}$ under Constraint (13). Substitute the expression of $\tau_{13} ; e_{1}^{(1)}=e_{1}$ satisfy $B_{1}^{\prime}\left(e_{1}\right)+B_{3}^{\prime}\left(e_{1}+e_{2}+e_{3}\right)=C_{1}^{\prime}\left(e_{1}\right)$. By symmetry, the optimal offer of 3 is $e_{1}^{(3)}=e_{1}=e_{1}^{(1)}$. Also by symmetry, the optimal offer of 2 to 3 $\left(o_{2}^{(2)}\right)$ maximizes his payoff under Constraint (15). It yields the optimal offer $e_{2}^{(2)}=e_{2}$ that satisfies $B_{2}^{\prime}\left(e_{1}+e_{2}\right)+B_{3}^{\prime}\left(e_{1}+e_{2}+e_{3}\right)=C_{2}^{\prime}\left(e_{2}\right)$. The optimal effort of agent 3 is given by $B_{3}^{\prime}\left(e_{1}+e_{2}+e_{3}\right)=$ 
$C_{3}^{\prime}\left(e_{3}\right)$. The associated equilibrium transfers $\tau_{13}=\tau_{13}^{(3)}$ and $\tau_{23}=\tau_{23}^{(3)}$, when 3 is the proposer in the two rounds, are:

$$
\begin{aligned}
& \tau_{13}=-\frac{\left(1-\delta_{1}\right)}{\left(1-\delta_{1} \delta_{3}\right)}\left(V_{1}^{*}-\pi_{1}^{n c}\right)+\frac{\delta_{1}\left(1-\delta_{3}\right)}{\left(1-\delta_{1} \delta_{3}\right)}\left(V_{3}^{*}-\pi_{3}^{n c}\right)-\frac{\delta_{1}\left(1-\delta_{3}\right)}{\left(1-\delta_{1} \delta_{3}\right)} \tau_{23} \\
& \tau_{23}=-\frac{\left(1-\delta_{2}\right)}{\left(1-\delta_{2} \delta_{3}\right)}\left(V_{2}^{*}-\pi_{2}^{n c}\right)+\frac{\delta_{2}\left(1-\delta_{3}\right)}{\left(1-\delta_{2} \delta_{3}\right)}\left(V_{3}^{*}-\pi_{3}^{n c}\right)-\frac{\delta_{2}\left(1-\delta_{3}\right)}{\left(1-\delta_{2} \delta_{3}\right)} \tau_{13}
\end{aligned}
$$

Solving returns:

$$
\begin{aligned}
& \tau_{13}^{*}=-\frac{\left(1-\delta_{1}\right)\left(1-\delta_{2} \delta_{3}\right)}{\eta}\left(V_{1}^{*}-\pi_{1}^{n c}\right)+\frac{\delta_{1}\left(1-\delta_{2}\right)\left(1-\delta_{3}\right)}{\eta}\left(V_{2}^{*}-\pi_{2}^{n c}+V_{3}^{*}-\pi_{3}^{n c}\right) \\
& \tau_{23}^{*}=-\frac{\left(1-\delta_{2}\right)\left(1-\delta_{1} \delta_{3}\right)}{\eta}\left(V_{2}^{*}-\pi_{2}^{n c}\right)+\frac{\delta_{2}\left(1-\delta_{1}\right)\left(1-\delta_{3}\right)}{\eta}\left(V_{1}^{*}-\pi_{1}^{n c}+V_{3}^{*}-\pi_{3}^{n c}\right)
\end{aligned}
$$

with $\eta=\left(1-\delta_{1} \delta_{3}\right)\left(1-\delta_{2} \delta_{3}\right)-\delta_{1} \delta_{2}\left(1-\delta_{3}\right)^{2}>0$. Equilibrium payoffs are given in the proposition.

\subsection{Proposition 3}

The same method applies when 2 bargains with 1 over $\left(e_{1}, \tau_{12}\right)$ and with 3 over $\left(e_{2}, \tau_{23}\right)$. The SPE offers solve the two systems of two indifference conditions between 1 and 2:

$$
\begin{gathered}
V_{1}\left(e_{1}^{(2)}\right)+\tau_{12}^{(2)}=\left(1-\delta_{1}\right) \pi_{1}^{n c}+\delta_{1}\left(V_{1}\left(e_{1}^{(1)}\right)+\tau_{12}^{(1)}\right) \\
V_{2}\left(e_{1}^{(1)}, e_{2}\right)+\tau_{23}-\tau_{12}^{(1)}=\left(1-\delta_{2}\right) \pi_{2}^{n c}+\delta_{2}\left(V_{2}\left(e_{1}^{(2)}, e_{2}\right)+\tau_{23}-\tau_{12}^{(2)}\right)
\end{gathered}
$$

and between 2 and 3 :

$$
\begin{gathered}
V_{2}\left(e_{1}, e_{2}^{(3)}\right)+\tau_{23}^{(3)}-\tau_{12}=\left(1-\delta_{2}\right) \pi_{2}^{n c}+\delta_{2}\left(V_{2}\left(e_{1}, e_{2}^{(2)}\right)+\tau_{23}^{(2)}-\tau_{12}\right) \\
V_{3}\left(e_{1}, e_{2}^{(2)}, e_{3}\right)-\tau_{23}^{(2)}=\left(1-\delta_{3}\right) \pi_{3}^{n c}+\delta_{3}\left(V_{3}\left(e_{1}, e_{2}^{(3)}, e_{3}\right)-\tau_{23}^{(3)}\right)
\end{gathered}
$$

Optimal equilibrium transfers when agent 2 is the first proposer are:

$$
\begin{aligned}
& \tau_{12}^{*}=\frac{\left(1-\delta_{1}\right)\left(1-\delta_{2} \delta_{3}\right)}{\phi}\left(V_{1}^{*}-\pi_{1}^{n c}\right)+\left(\frac{\delta_{1}\left(1-\delta_{2}\right)\left(1-\delta_{3}\right)}{\phi}\right)\left(V_{2}^{*}-\pi_{2}^{n c}+V_{3}^{*}-\pi_{3}^{n c}\right) \\
& \tau_{23}^{*}=-\frac{\left(1-\delta_{1}\right)\left(1-\delta_{2}\right) \delta_{3}}{\phi}\left(V_{1}^{*}-\pi_{1}^{n c}+V_{2}^{*}-\pi_{2}^{n c}\right)+\frac{\left(1-\delta_{3}\right)\left(1-\delta_{1} \delta_{2}\right)}{\phi}\left(V_{3}^{*}-\pi_{3}^{n c}\right)
\end{aligned}
$$

with $\phi=\left(1-\delta_{1} \delta_{2}\right)\left(1-\delta_{2} \delta_{3}\right)-\delta_{1} \delta_{3}\left(1-\delta_{2}\right)^{2}>0$. Optimal emissions and payoffs are in the proposition.

\subsection{Results}

Results are summarized in the following table: 
Table 2. Efforts (in $\bar{e}$ ) and payoffs (in $b \bar{e}^{2}$ ) in the cooperative and non-cooperative cases. $\gamma=c / b$ and $\bar{e}=a / b$.

\begin{tabular}{lcc}
\hline & Coop & Non-Coop \\
\hline$e_{1}$ & $\frac{1+4 \gamma+3 \gamma^{2}}{5 \gamma+6 \gamma^{2}+\gamma^{3}+1}$ & $\frac{1}{1+\gamma}$ \\
\hline$e_{2}$ & $\frac{\gamma+2 \gamma^{2}}{5 \gamma+6 \gamma^{2}+\gamma^{3}+1}$ & $\frac{\gamma}{(\gamma+1)^{2}}$ \\
\hline$e_{3}$ & $\frac{\gamma^{2}}{5 \gamma+6 \gamma^{2}+\gamma^{3}+1}$ & $\frac{\gamma^{2}}{(\gamma+1)^{3}}$ \\
\hline$\pi_{1}$ & $\frac{\left(3 \gamma^{2}+4 \gamma+1\right)\left(-\gamma^{3}+5 \gamma^{2}+5 \gamma+1\right)}{2\left(\gamma^{3}+6 \gamma^{2}+5 \gamma+1\right)^{2}}$ & $\frac{1}{2(\gamma+1)}$ \\
\hline$\pi_{2}$ & $\frac{6 \gamma^{2}+5 \gamma+1}{2\left(\gamma^{3}+6 \gamma^{2}+5 \gamma+1\right)}$ & $\frac{\left(3 \gamma^{3}+6 \gamma^{2}+4 \gamma+1\right)}{2(\gamma+1)^{4}}$ \\
\hline$\pi_{3}$ & $\frac{\left(11 \gamma^{5}+46 \gamma^{4}+62 \gamma^{3}+37 \gamma^{2}+10 \gamma+1\right)}{2\left(\gamma^{3}+6 \gamma^{2}+5 \gamma+1\right)^{2}}$ & $\frac{\left(5 \gamma^{5}+15 \gamma^{4}+20 \gamma^{3}+15 \gamma^{2}+6 \gamma+1\right)}{2(\gamma+1)^{6}}$ \\
\hline
\end{tabular}

When $\gamma=0$ (for $c=0$ ), $e_{1}=\bar{e}$, while $e_{i}=0$ for $i=2,3$ and $\pi_{i}=(b / 2) \bar{e}^{2} \forall i$ in both cooperative and non-cooperative cases. It can be shown that $e_{1}$ decreases with $\gamma$, while $e_{i}=0$ for $i=2,3$ first increases, reaches a maximum and then decreases. Payoffs always decrease with $\gamma$. A low value of $\gamma$ means a low marginal cost and/or a high marginal benefit, implying higher efforts and payoffs. On the opposite side, a high value of $\gamma$ means a high marginal cost $(c)$ and/or a low marginal benefit $(b)$, implying lower efforts and payoffs. These patterns are the same for all of the negotiation protocols.

The negotiation procedures involving only two agents are summarized in the following table ${ }^{2}$ :

Table 3. Efforts (in $\bar{e}$ ) and payoffs (in $b \bar{e}^{2}$ ) in the two-by-two negotiation process.

\begin{tabular}{cccc}
\hline & $\mathbf{2} \leftrightarrow \mathbf{3}$ & $\mathbf{1} \leftrightarrow \mathbf{3}$ & $\mathbf{1} \leftrightarrow \mathbf{2}$ \\
\hline$e_{1}$ & $\frac{1}{1+\gamma}$ & $\frac{1+2 \gamma+2 \gamma^{2}}{3 \gamma+4 \gamma^{2}+\gamma^{3}+1}$ & $\frac{1+2 \gamma}{3 \gamma+\gamma^{2}+1}$ \\
\hline$e_{2}$ & $\frac{\gamma+2 \gamma^{2}}{4 \gamma+4 \gamma^{2}+\gamma^{3}+1}$ & $\frac{\gamma+\gamma^{2}}{3 \gamma+4 \gamma^{2}+\gamma^{3}+1}$ & $\frac{\gamma}{3 \gamma+\gamma^{2}+1}$ \\
\hline$e_{3}$ & $\frac{\gamma^{2}}{4 \gamma+4 \gamma^{2}+\gamma^{3}+1}$ & $\frac{\gamma^{2}}{3 \gamma+4 \gamma^{2}+\gamma^{3}+1}$ & $\frac{\gamma^{2}}{4 \gamma+4 \gamma^{2}+\gamma^{3}+1}$ \\
\hline$\pi_{1}-\pi_{1}^{n c}$ & 0 & $\frac{\gamma^{6}\left(\gamma^{4}+3 \gamma^{3}-2 \gamma-1\right)}{4(\gamma+1)^{5}\left(\gamma^{3}+4 \gamma^{2}+3 \gamma+1\right)^{2}}$ & $\frac{\gamma^{4}}{4(\gamma+1)^{3}\left(\gamma^{2}+3 \gamma+1\right)}$ \\
\hline$\pi_{2}-\pi_{2}^{n c}$ & $\frac{\gamma^{6}}{4(\gamma+1)^{5}\left(\gamma^{2}+3 \gamma+1\right)}$ & $\frac{\gamma^{5}\left(2 \gamma^{3}+7 \gamma^{2}+6 \gamma+2\right)}{2(\gamma+1)^{3}\left(\gamma^{3}+4 \gamma^{2}+3 \gamma+1\right)^{2}}$ & $\pi_{1}-\pi_{1}^{n c}$ \\
\hline$\pi_{3}-\pi_{3}^{n c}$ & $\pi_{2}-\pi_{2}^{n c}$ & $\pi_{1}-\pi_{1}^{n c}$ & $\frac{\left(2 \gamma^{2}+5 \gamma+2\right) \gamma^{6}}{2(\gamma+1)^{5}\left(\gamma^{2}+3 \gamma+1\right)^{2}}$ \\
\hline
\end{tabular}

2 The created surplus in the negotiation between 1 and 3 is positive for $\gamma>0.89$. 
Negotiation protocols consisting in two bilateral negotiations are summarized in the following table:

Table 4. Efforts (in $\bar{e}$ ) and payoffs (in $\left.b \bar{e}^{2}\right)$ in double negotiation.

\begin{tabular}{ccc}
\hline & $\mathbf{3} \longleftrightarrow \mathbf{1 , 2}$ & $\mathbf{2} \longleftrightarrow \mathbf{1 , 3}$ \\
$e_{1}$ & $\frac{1+3 \gamma+2 \gamma^{2}}{4 \gamma+5 \gamma^{2}+\gamma^{3}+1}$ & $\frac{1+4 \gamma+2 \gamma^{2}}{5 \gamma+5 \gamma^{2}+\gamma^{3}+1}$ \\
\hline$e_{2}$ & $\frac{\gamma+2 \gamma^{2}}{4 \gamma+5 \gamma^{2}+\gamma^{3}+1}$ & $\frac{\gamma+2 \gamma^{2}}{5 \gamma+5 \gamma^{2}+\gamma^{3}+1}$ \\
\hline$e_{3}$ & $\frac{\gamma^{2}}{4 \gamma+5 \gamma^{2}+\gamma^{3}+1}$ & $\frac{\gamma^{2}}{5 \gamma+5 \gamma^{2}+\gamma^{3}+1}$ \\
\hline$\pi_{i}-\pi_{i}^{n c}$ & $\frac{\left(4 \gamma^{5}+23 \gamma^{4}+38 \gamma^{3}+30 \gamma^{2}+12 \gamma+2\right) \gamma^{5}}{6(\gamma+1)^{5}\left(\gamma^{3}+5 \gamma^{2}+4 \gamma+1\right)^{2}}$ & $\frac{\left(4 \gamma^{4}+17 \gamma^{3}+15 \gamma^{2}+7 \gamma+1\right) \gamma^{4}}{6(\gamma+1)^{5}\left(\gamma^{2}+4 \gamma+1\right)^{2}}$ \\
\hline
\end{tabular}

Comparing the different outcomes gives in terms of individuals ${ }^{3}$ and aggregate efforts:

- $e_{1}^{c}>e_{1}^{\{1 \longleftrightarrow 2\}}>e_{1}^{\{2 \longleftrightarrow 1,3\}}>e_{1}^{\{1 \longleftrightarrow 3\}}>e_{1}^{\{3 \longleftrightarrow 1,2\}}>e_{1}^{n c}=e_{1}^{\{2 \longleftrightarrow 3\}}$

- $e_{2}^{\{2 \longleftrightarrow 3\}}>e_{2}^{\{3 \longleftrightarrow 1,2\}}>e_{2}^{\{2 \longleftrightarrow 1,3\}}>e_{2}^{c}>e_{2}^{n c}>e_{2}^{\{1 \longleftrightarrow 3\}}>e_{2}^{\{1 \longleftrightarrow 2\}}$

- $e_{3}^{n c}>e_{3}^{\{1 \longleftrightarrow 3\}}>e_{3}^{\{2 \longleftrightarrow 3\}}=e_{3}^{\{1 \longleftrightarrow 2\}}>e_{3}^{\{3 \longleftrightarrow 1,2\}}>e_{3}^{\{2 \longleftrightarrow 1,3\}}>e_{3}^{c}$

- $\sum_{i} e_{i}^{c}>\sum_{i} e_{i}^{\{2 \longleftrightarrow 1,3\}}>\sum_{i} e_{i}^{\{3 \longleftrightarrow 1,2\}}>\sum_{i} e_{i}^{\{1 \longleftrightarrow 2\}}=\sum_{i} e_{i}^{\{2 \longleftrightarrow 3\}}>\sum_{i} e_{i}^{\{1 \longleftrightarrow 3\}}>\sum_{i} e_{i}^{n c}$

The individual and aggregate payoffs are:

- $\pi_{1}^{\{2 \longleftrightarrow 1,3\}}>\pi_{1}^{\{3 \longleftrightarrow 1,2\}}>\pi_{1}^{\{1 \longleftrightarrow 2\}}>\pi_{1}^{\{1 \longleftrightarrow 3\}}>\pi_{1}^{n c}=\pi_{2}^{\{2 \longleftrightarrow 3\}}>\pi_{1}^{c}$

- $\pi_{2}^{c}>\pi_{2}^{\{1 \longleftrightarrow 3\}}=\pi_{2}^{F}>\pi_{2}^{\{2 \longleftrightarrow 1,3\}}>\pi_{2}^{\{3 \longleftrightarrow 1,2\}}>\pi_{2}^{\{1 \longleftrightarrow 2\}}>\pi_{2}^{\{2 \longleftrightarrow 3\}}>\pi_{2}^{n c}$

- $\pi_{3}^{c}>\pi_{3}^{\{1 \longleftrightarrow 2\}}=\pi_{3}^{F}>\pi_{3}^{\{2 \longleftrightarrow 1,3\}}>\pi_{3}^{\{3 \longleftrightarrow 1,2\}}>\pi_{3}^{\{2 \longleftrightarrow 3\}}>\pi_{3}^{\{1 \longleftrightarrow 3\}}>\pi_{3}^{n c}$

- $\sum_{i} \pi_{i}^{c}>\sum_{i} \pi_{i}^{\{2 \longleftrightarrow 1,3\}}>\sum_{i} \pi_{i}^{\{3 \longleftrightarrow 1,2\}}>\sum_{i} \pi_{i}^{\{1 \longleftrightarrow 2\}}>\sum \pi_{i}^{\{1 \longleftrightarrow 3\}}>\sum \pi_{i}^{\{2 \longleftrightarrow 3\}}>\sum_{i} \pi_{i}^{n c}$

\section{Acknowledgments}

This study received funding from the French ministry of Ecology, Sustainable Development and Energy, in the "Eaux et Territoires" research program of 10/16/2010, convention No. 2100170383 and the French National Research Agency as part of the project, ADAPTEAU (ANR-11-CEPL-008), in the frame of the Cluster of Excellence COTE (ANR-10-LABX-45). For agent 2, the rank holds for $\gamma>\gamma^{*}=\frac{1}{2}(1+\sqrt{5})$ and for $\gamma \geq 1: e_{2}^{\{1,3 \longleftrightarrow 2\}} \geq e_{2}^{n c}$, for $\gamma \geq 0.815: e_{2}^{c} \geq e_{2}^{\{1 \longleftrightarrow 3\}}$
and for $\gamma \geq 0.52: e_{2}^{\{1,3 \longleftrightarrow 2\}} \geq e_{2}^{\{1 \longleftrightarrow 3\}}$ 


\section{Conflicts of Interest}

The authors declare no conflict of interest.

\section{References}

1. Hirshleifer, J. From weakest-link to best-shot: The voluntary provision of public goods. Public Choice 1983, 41, 371-386.

2. Rubinstein, A. Perfect equilibrium in a bargaining model. Econometrica 1982, 50, 97-109.

3. Carraro, C.; Siniscalco, D. Strategies for the international protection of the environment. J. Public Econ. 1993, 52, 309-328.

4. Barrett, S. Self-enforcing international environmental agreements. Oxf. Econ. Pap. 1994, 46, 878-894.

5. Finus, M. Stability and design of international environmental agreements: The case of global and transboundary pollution. International Yearbook of Environmental and Resource Economics; Folmer, H., Tietenberg, T., Eds.; Edward Elgar: Cheltenham, UK, 2003; pp. 82-158.

6. Ambec, S.; Sprumont, Y. Sharing a river. J. Econ. Theory 2002, 107, 453-462.

7. Beal, S.; Ghintran, A.; Remila, E.; Solal, P. The river sharing problem: A survey. Int. Game Theory Rev. 2013, 15, doi:10.1142/S0219198913400161.

8. Carraro, C.; Marchiori, C.; Sgobbi, A. Negotiation on water: Insights from non-cooperative bargaining theory. Environ. Dev. Econ. 2007, 12, 329-349.

9. Wang, Y. Trading water along a river. Math. Soc. Sci. 2011, 61, 124-130.

10. Houba, H. Computing alternating offers and water prices in bilateral river basin management. Int. Game Theory Rev. 2008, 10, 257-278.

11. Houba, H.; van der Laan, G.; Zeng, Y. Asymmetric Nash solutions in the river sharing problem. Tinbergen Inst. Discuss. Paper 2013, 51, 28 pages.

12. Sutton, J. Non-cooperative bargaining theory: An introduction. Rev. Econ. Stud. 1986, 53, 709-724.

13. Muthoo, A. Bargaining Theory with Applications; Cambridge University Press: Cambridge, UK, 1999.

(c) 2014 by the authors; licensee MDPI, Basel, Switzerland. This article is an open access article distributed under the terms and conditions of the Creative Commons Attribution license (http://creativecommons.org/licenses/by/3.0/). 